\title{
大氣波動の減衰と䓢期に及㾏寸粘性の影響 (第一報)
}

\section{村 濑 信 夫}

\section{緒言}

大氣の微振動に就ては多くの學者が研究發表(1)して居るが粘性がどんな影響をなすか餘り研究さ れて居ない樣である.著者はての影響を調べをい。

先 $x$ 軸を水平飞 $z$ 軸を鉛直にとり平衡の時は

$$
\frac{d p_{0}}{d z}=-\rho_{0} g
$$

氣體方程式之して㹥次の式を用ひる。

$$
p_{0}=\rho_{0} R T
$$

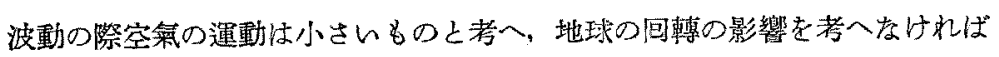

$$
\rho \frac{\partial u}{\partial t}=-\frac{\partial p}{\partial x}+\rho \nu \nabla u, \quad \rho \frac{\partial w}{\partial t}=-\frac{\partial p}{\partial z}+\rho \nu \nabla w-g
$$

連續の式は

$$
\frac{d \rho}{d t}+\rho\left(\frac{\partial u}{\partial x}+\frac{\partial w}{\partial z}\right)=0
$$

波動による空氣の狀態綎化は斷熱的であると假定すれば

$$
\frac{d p}{d t}=c^{2} \frac{d \rho}{d t}, \quad c^{2}=\gamma \frac{p_{0}}{\rho_{0}}=\gamma R T_{0}
$$

借 $p=p_{0}+p^{\prime}, \rho=\rho_{0}+\rho^{\prime}$ 乙むき $p^{\prime}, \rho^{\prime}$ は小さいから二次以上忙無視する。然るとを $(3),(4),(5)$ は次の樣沈なる。

$$
\begin{gathered}
\rho_{0} \frac{\partial u}{\partial t}=-\frac{\partial p^{\prime}}{\partial x}+\rho_{0} \nu \nabla u, \quad \rho_{0} \frac{\partial w}{\partial t}=-\frac{\partial p^{\prime}}{\partial z}+\rho_{0} \nu \nabla w-g \rho^{\prime} \\
\frac{\partial \rho^{\prime}}{\partial t}+w \frac{\partial \rho_{0}}{\partial z}=-\rho_{0} \chi \\
\frac{\partial p^{\prime}}{\partial t}-\rho_{0} g w=-r p_{0} \chi
\end{gathered}
$$

但し

$$
\chi=\frac{\partial u}{\partial x}+\frac{\partial w}{\partial z}
$$

$(4)^{\prime},(5)^{\prime}$ を(3)'に入れると

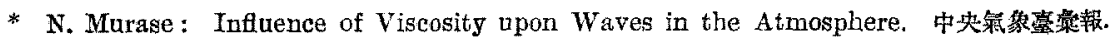

(1) Kwaniti Koenuma: Some Problems of Waves Propagating in the Atmosphere. 戴像學㷋石, p. 421 . 


$$
\left.\begin{array}{l}
\left(\frac{\partial^{2}}{\partial t^{2}}-\frac{\partial}{\partial t} \nu \nabla\right) u=c^{2} \frac{\partial \chi}{\partial x}-g \frac{\partial w}{\partial x} \\
\left(\frac{\partial^{2}}{\partial t^{2}}-\frac{\partial}{\partial t} \nu \nabla\right) w=c^{2} \frac{\partial \chi}{\partial z}-g \frac{\partial w}{\partial z}-g(\gamma-1)
\end{array}\right\}
$$

上式から

$$
\left(\frac{\partial^{2}}{\partial t^{2}}-\frac{\partial}{\partial t} \nu \nabla\right) \chi=c^{2} \nabla \chi+\left(\frac{d c^{2}}{\partial z}-g \gamma\right) \frac{\partial \chi}{\partial z}+g \frac{\partial \eta}{\partial x}
$$

幽K

$$
\eta=\frac{\partial u}{\partial z}-\frac{\partial w}{\partial x}
$$

(6) ๖り

$$
\left.\left(\frac{\partial^{2}}{\partial t^{2}}-\frac{\partial}{\partial t} \nu \nabla\right) \eta=\left[\frac{\partial^{2} c}{\partial z^{2}}+g(\gamma-1)\right)\right] \frac{\partial X}{\partial z}
$$

(6) と (8) を組合せると

$$
\begin{aligned}
\left(\frac{\partial^{2}}{\partial t^{2}}-\frac{\partial}{\partial t} \nu \nabla\right)^{2}= & c^{2} \nabla\left(\frac{\partial^{2}}{\partial t^{2}}-\frac{\partial}{\partial t} \nu \nabla\right) \chi+\left(\frac{d c^{2}}{d z}-g \gamma\right)\left(\frac{\partial^{2}}{\partial t^{2}}-\frac{\partial}{\partial t} \nu \nabla\right) \chi \\
& +g\left[\frac{d c^{2}}{d z}+g(\gamma-1)\right] \frac{\partial^{2} \chi}{\partial x^{2}}
\end{aligned}
$$

先つ最初，極めて簡單な場合を考へ上う。

氣溫は一定で波動による空氣の狀態變化は等溫であるとする.然る時は $(9)$ 式は簡單になる $\left(T_{0}\right.$ を $T$ と書くこと炡する)

$$
\left(\frac{\partial^{2}}{\partial t^{2}}-\frac{\partial}{\partial t} \nu \nabla\right)\left(\frac{\partial^{2}}{\partial t^{2}}-\frac{\partial}{\partial t} \nu \nabla-R T \nabla+g \frac{\partial}{\partial z}\right) \chi=0
$$

故k

$$
\frac{\partial^{2}}{\partial t^{2}}-\frac{\partial}{\partial t} \nu \nabla-R T \nabla+g \frac{\partial \chi}{\partial z}=0
$$

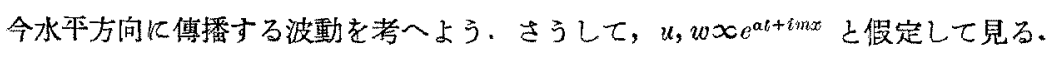

(11) より

$$
\frac{a^{2} \chi}{d z^{2}}-\frac{g}{R T+\alpha \nu} \frac{d \chi}{d z}-\left(m^{2}+\frac{\alpha^{2}}{R T+\alpha \nu}\right) \chi=0
$$

故保

$$
\chi=\left(A e^{\beta_{1} z}+B e^{\beta_{2} z}\right) e^{a t+\varepsilon m: x}
$$

此處に

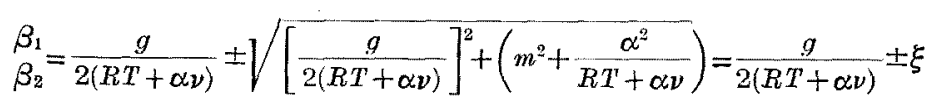

但し

$$
\xi^{2}=\left[\frac{g}{2(R T+\alpha \nu)}\right]^{2}+\left(m^{2}+\frac{\alpha^{2}}{R T+\alpha \nu}\right)
$$

借 $w=\left(a A e^{\beta_{2} z}+b B e^{\beta 2 z}\right) e^{a t+t m x}$ とおいて (6) より $a, b$ を求めると

$$
a=\frac{R T \beta_{1}}{\alpha^{2}-\alpha \nu \beta_{1}^{2}+\alpha \nu m^{2}+g \beta_{1}^{2}} \quad b=\frac{R T \beta_{2}}{\alpha^{2}-\alpha \nu \beta_{2}^{2}+\alpha \nu m^{2}+g \beta_{2}}
$$

今境界條件として次の樣に取る。

$$
\begin{gathered}
{[w]_{z=0}=0 \quad[\chi]_{z=H}=0} \\
-37-
\end{gathered}
$$


(17) より

$$
a A+b B=0, \quad A e^{f_{1} H}+B e^{Q_{2} H}=0
$$

(18)より $A, B$ 老消去し， $a, b$ の值老代入すると

$$
\frac{\beta_{2} e^{\beta_{1} H}}{\alpha^{3}-\alpha \nu \beta_{1}{ }^{3}+\alpha \nu m^{2}+g \beta_{1}}=\frac{\beta_{1} e^{\beta_{2} I T}}{\alpha^{2}-\alpha \nu \beta_{2}^{2}+\alpha \nu m^{2}+g \beta_{2}}
$$

上式に $\beta_{1}, \beta_{2}$ の值を代入し整頓すると

$$
\xi \operatorname{coth} \xi H=-\frac{g m^{2}}{R T \alpha^{2}}(R T+\alpha \nu)-\frac{g}{2(R T+\alpha \nu)}
$$

今若し大氣の上界 $H$ を相當大きくとると $\operatorname{coth} \xi H \div 1$ となり

$$
\xi=-\frac{g m^{2}}{R T \alpha^{2}}(R T+\alpha \nu)-\frac{g}{2(R T+\alpha \nu)}
$$

(21) と (15) 上り $\xi$ を消去し整理すると

$$
\begin{aligned}
& \alpha^{6}+m^{2} \nu \alpha^{5}+R T^{2} m^{2} \alpha^{4}-\frac{g^{2} m^{2} \nu}{R^{2} T^{2}}\left(R T^{\prime}+m^{2} \nu^{2}\right) \alpha^{3} \\
& -\frac{g^{9} m^{2}}{R T^{\prime}}\left(R T+3 m^{2} \nu^{2}\right) \alpha^{2}-3 g^{2} m^{4} \nu \alpha-g^{2} m^{4} R T^{\prime}=0
\end{aligned}
$$

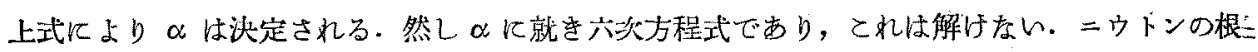
の近似値を求める公式を應用し，第一近似值を出して見る.

上式江 $\nu=0$ 上小げ゙

$$
\begin{aligned}
& \left(\alpha^{2}+m^{2} R T,\left(\alpha^{4}-g^{2} m^{2}\right)=0\right. \\
& \alpha=i m \sqrt{R T}, \quad \alpha=i \sqrt{g^{m}}
\end{aligned}
$$

從つて

てれは肥涩先生の求められを值に $\gamma=1$ としたものに同一である.

ニウトンの方法によると， $f(\alpha)=0$ の一つの根の近似值を $\alpha_{1}$ とすれば

は次第に正しき根汇近づく。

$$
\alpha_{2}=\alpha_{1}-\frac{f\left(\alpha_{1}\right)}{f^{\prime}\left(\alpha_{1}\right)}, \quad \alpha_{3}=\alpha_{2}-\frac{f\left(\alpha_{2}\right)}{f^{\prime}\left(\alpha_{2}\right)},
$$

$\alpha_{1}$ として (21) 式の根を用ひる. 然る時

$$
f(i m R T)=i X+Y \quad f^{\prime}(i m R T)=i X^{\prime}+Y^{\prime}
$$

となつたとする，玆に $X, Y, X^{\prime}, Y^{\prime}$ は头々次の值を有する.

故炕

$$
\begin{gathered}
X=m^{5} \nu\left(m^{2} R^{2} \eta^{2} \sqrt{R T}-\frac{g^{2} m^{2} \nu^{2}}{\sqrt{R T}}-2 g^{2} \sqrt{R T}\right), \quad Y=3 g^{2} m^{6} \nu^{2} \\
X^{\prime}=m^{6} \nu\left(5 R^{2} I^{2}+\frac{3 g^{2} \nu^{2}}{R T}\right) \\
X^{\prime}=2 m^{3}\left(m^{2} K^{2} T^{\prime 2} \sqrt{R T}-3 \frac{g^{2} m^{2} v^{2}}{\sqrt{R T}}-g^{2} \sqrt{R T}\right) \\
\alpha=i\left(m \sqrt{R T}-\frac{X X^{\prime}-X^{\prime} Y}{X^{\prime 2}+Y^{\prime 2}}\right)-\frac{X X^{\prime}+Y Y^{\prime}}{X^{\prime 2}+Y^{\prime 2}} \\
-38-
\end{gathered}
$$


從つて

$$
u w X \infty e^{i\left(m \sqrt{k T}-\frac{X Y^{\prime}-X^{\prime} T}{X^{\prime}+Y^{\prime 2}}\right) t-\frac{X X^{\prime}+Y^{\prime}}{X^{\prime} 8+Y^{\prime} 2} t+\{m s}
$$

上式より明らかなる樣に $\frac{X X^{\prime}+Y Y^{\prime}}{X^{\prime 2}+Y^{\prime 2}}$ は粘性による振幅の減衰度を示し， $\frac{X Y^{\prime}-X^{\prime} Y}{X^{\prime 2}+Y^{\prime 2}}$ は粘性によ る振動週期の變り方を表して居る．今ての粘性の影響を調べて見よう。

(26) ๖り

$$
\begin{gathered}
X X^{\prime}+Y Y^{\prime}=m^{9} \nu^{2}\left[\left(5 R^{4} T^{4} \sqrt{R T}-2 g^{2} R T^{\prime} \sqrt{R T} \nu^{2}-3 \frac{g^{4} \nu^{4}}{R T^{\prime} \sqrt{R T}}\right) m^{4}\right. \\
\left.-4\left(g^{2} R^{2} T^{2} \sqrt{R T}+6 \frac{g^{4} \nu^{2}}{\sqrt{R T}}\right) m^{2}-6 g^{4} R T\right] \\
X Y^{\prime}-X^{\prime} Y=m^{8} \nu\left[\left(2 R^{5} T^{5}-23 g^{2} \nu^{2} R^{2} T^{2}-3 \frac{g^{4} \nu^{4}}{R T^{\prime}}\right) m^{4}\right. \\
\left.-2\left(3 g^{2} R^{3} T^{3}-7 g^{4} \nu^{2}\right) m^{2}+4 g^{4} R T^{\prime}\right] \\
X^{\prime 2}+Y^{\prime 2}=m^{6}\left[\left(25 R^{4} T^{4} \nu^{2}+9 \frac{g^{4} \nu^{6}}{R^{2} T^{2}}+30 g^{2} R T^{4} \nu^{4}\right) m^{6}\right. \\
\left.+4\left(R^{4} T^{5}+9 \frac{g^{4} \nu^{4}}{R T}-6 R^{2} T^{2} \nu^{2}\right) m^{4}+8\left(3 g^{4} \nu^{2}-g^{2} R^{3} I^{3}\right) m^{2}+4 g^{4} R T\right]
\end{gathered}
$$

(29) と (31)，(28) と（31）とを組合せ

$$
\begin{aligned}
& \frac{X X^{\prime}+Y Y^{\prime}}{\bar{X}^{\prime 2}+Y^{\prime 2}}=m^{3} \nu^{2}\left[\left(5 R^{4} T^{4} \sqrt{R T}-2 g^{2} R T \sqrt{R T} \nu^{2}-3 \frac{g^{4} \nu^{4}}{R T \sqrt{R T}}\right) m^{4}-4\left(g^{2} R^{2} T^{2} \sqrt{R T}+6 \frac{g^{4} \nu^{2}}{\sqrt{R T}}\right) m^{2}-6 g^{4} R T\right] \\
& \div\left[\left(25 R^{4} T^{4} \nu^{2}+9 \frac{g^{4} \nu^{6}}{R^{2} T^{2}}+30 g^{2} R T^{\prime} \nu^{4}\right) m^{9}+4\left(R^{5} T^{5}+9 \frac{g^{4} \nu^{4}}{R T^{T}}-6 R^{2} T^{2} \nu^{2}\right) m^{ \pm}\right. \\
& \left.+8\left(3 g^{4} \nu^{2}-g^{2} R^{3} T^{3}\right) m^{2}+4 g^{4} R T\right] \\
& \frac{X Y^{\prime}-X^{\prime} Y}{X^{\prime 2}+\bar{Y}^{\prime 2}}=m^{2} \nu\left[\left(2 R^{5} T^{5}-23 g^{2} \nu^{2} R^{2} T^{2}-3 \frac{g^{4} \nu^{4}}{R T}\right) m^{4}-2\left(3 g^{2} R^{3} T^{3}-7 g^{4} \nu^{9}\right) m^{2}+4 g^{4} R T\right] \\
& \div\left[\left(25 R^{4} T^{4} \nu^{2}+9 \frac{g^{4} \nu^{6}}{R^{2} T^{2}}+30 g^{2} R T \nu^{4}\right) m^{6}+4\left(R^{5} T^{5}+9 \frac{g^{4} \nu^{4}}{R T}-6 R^{2} T^{3} \nu^{2}\right) m^{4}\right. \\
& \left.+8\left(3 g^{4} \nu^{2}-g^{2} R^{3} T^{3}\right) m^{2}+4 g^{4} R T\right]
\end{aligned}
$$

(32),(33) が所要のものである。

波動の安定不安定を論和るに位

を調べればよい。

$$
\begin{array}{ll}
X X^{\prime}+Y Y^{\prime}>0 & \text { 減変振動 } \\
X X^{\prime}+Y Y^{\prime}=0 & \text { 安定振動 } \\
X X^{\prime}+Y Y^{\prime}<0 & \text { 不安定振動 }
\end{array}
$$

(36) 加方

$$
\begin{gathered}
\left(5 R^{4} T^{4} \sqrt{R T}-2 g^{2} R T \sqrt{R T^{2}} \nu^{2}-3 \frac{g^{4} \nu^{4}}{R T \sqrt{R T}}\right) m^{4}-4\left(g^{2} R^{2} T^{2} \sqrt{R T}+6 \frac{g^{4} \nu^{2}}{\sqrt{R T}}\right) m^{2}-6 g^{4} R T^{\prime}=0 \\
-39-
\end{gathered}
$$




$$
\begin{aligned}
m^{2} & =\frac{2\left(g^{2} R^{2} T^{12} \sqrt{R T}+6 \frac{g^{4} \nu^{2}}{\sqrt{R T}}\right) \pm \sqrt{34 g^{1} R^{5} T^{5}+46 g^{6} R^{2} T^{2} \nu^{2}+126 \frac{g^{8} \nu^{4}}{R T}}}{5 R^{4} T^{4} \sqrt{R T}-2 g^{2} R T \sqrt{R T} \nu^{2}-3 \frac{g^{4} \nu^{4}}{R \nu^{4} \sqrt{R T}}} \\
& =\frac{2\left(g^{2}+6 \frac{g^{4} \nu^{2}}{R^{3} \eta^{73}}\right) \pm \sqrt{34} g^{2} \sqrt{1+\frac{23}{17} \frac{g^{2} \nu^{2}}{R^{3} T^{3}}+\frac{63}{17} \frac{g^{4} \nu^{4}}{R^{6} T^{6}}}}{5 R^{2} T^{4}-2 \frac{g^{2} \nu^{2}}{R T^{4}}-3 \frac{g^{4} \nu^{4}}{R^{4} T^{4}}}
\end{aligned}
$$

然るに大氣の粘性は大きく見積つて $10^{8}$ 之看做して, 微少項を省略すると(波動炕分子粘性が 作用すると考へられるがこれ程大きく見糔つても)

$$
m^{2} \div \frac{7.83 g^{2}}{5 R^{2} \eta^{2}} \div 1.57 \frac{g^{2}}{R^{2} \eta^{2}}
$$

故炕

$$
m=1 \cdot \dot{25}-\frac{g}{R T}
$$

仍つて波長入社

$$
\lambda=40 \mathrm{~km}
$$

これは柾密波であるが音波之は考へられない．從つて，40 km の波は安定で規律正しい振動であ る. $40 \mathrm{~km}$ 以下の波長の波は減哀振動ですり $40 \mathrm{~km}$ 以上のは不安定でする.減衰發散の程度は一

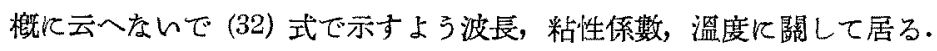

今特別の場合につを粘性の影響を出す，曋樂の如きは振動數 300〜500 位までである. それ故 波長 $1.00 \sim 0.60 \mathrm{~m}$ 位である.

今 $m=10^{-2}$ 〜 $10^{-1}$ 位之看做せ价 (33) の微小項を省略すると

$$
\frac{X X^{\prime}+Y Y^{\prime}}{\bar{X}^{\prime 2}+Y^{\prime 2}} \div \frac{5 m^{8} \nu^{2} \sqrt{R T}}{25 \nu^{2} m^{2}+4 R T}
$$

とおける.若し分子粘性しかないよすれば $\nu^{2} m^{2}<R T$ なる故

$$
\frac{X X^{\prime}+Y Y^{\prime}}{\bar{X}^{\prime 2}+Y^{\prime 2}} \div \frac{5}{4} m^{3} \nu^{2} \times \frac{1}{\sqrt{R T}}
$$

即ち，波長の三乘根に逆比例し，粘性の二乘に 此例することになるが $\nu$ は非常に小さい故殆ど 減衰しをいてとになる. 若しvを普通考へられてるる重力粘性保数と考へる時は. (39)を用ひなく てはいけない.

\section{粘性の週期に及ぼす影響}

$$
\begin{gathered}
\frac{X Y^{\prime}-X^{\prime} Y}{X^{\prime 2}+Y^{\prime 2}}=m^{2} v\left[\left(2 R^{7} T^{5}-23 g^{2} R^{2} T^{2}-3 \frac{g^{4} \nu^{4}}{R T^{7}}\right) m^{4}-2\left(3 g^{2} R^{3} T^{3}-7 g^{4} \nu^{2}\right) m^{2}+4 g^{4} R T\right] \\
\quad \div\left[\left(25 R^{4} T^{14} \nu^{2}+g \frac{g^{4} \nu^{6}}{R^{2} T^{2}}+3 g^{2} R T^{4} \nu^{4}\right) m^{6}+4\left(R^{5} T^{5}+9 \frac{g^{4} \nu^{4}}{R T}-6 R^{2} T^{3} \nu^{2}\right) m^{4}\right. \\
\left.+8\left(3 g^{4} \nu^{2}-g^{3} R^{3} T^{3}\right) m^{2}+4 g^{4} R T\right] \\
-40-
\end{gathered}
$$


今粘性は小さいとして微小項省省略すると

$$
\frac{X Y^{\prime}-X^{\prime} Y}{X^{\prime 2}+Y^{\prime 2}} \div \frac{1}{2} m^{2} \nu \frac{m^{2} R^{2} T^{2}-3 g^{2}}{m^{2} R^{2} T^{2}-g^{2}}
$$

波長吕相當短かければ

$$
\frac{X Y^{\prime}-X^{\prime} Y}{X^{\prime 2}+Y^{\prime 2}}=\frac{1}{2} m^{2} \nu
$$

波長が相當長ければ

$$
\frac{X Y^{\prime}-X^{\prime} Y}{X^{\prime 2}+Y^{\prime 2}}=m^{2} \nu
$$

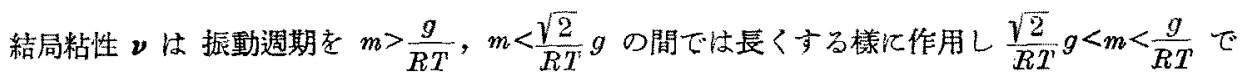
は短かくする样に衝くが若し音波の樣な波には分子粘性のみが作用すると考へればその影響忏極く

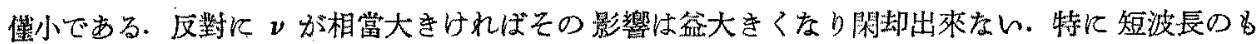

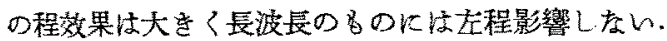

重力波の場合 郎方

$$
\alpha=i \sqrt{g m}
$$

$$
f(i \sqrt{g m})=i X+Y \quad f^{\prime}(i \sqrt{g m})=i X^{\prime}+Y^{\prime}
$$

となけば

$$
\begin{aligned}
& \mathrm{X}=\frac{g^{g} \sqrt{g m} m^{3} \nu}{R T}\left(g-2 m R T+\frac{m^{2} v^{2}}{R T}\right), \quad \mathrm{Y}=3 \frac{g^{3} m^{5} \nu^{2}}{R T} \\
& \mathrm{X}^{\prime}=2 g \sqrt{g m} m^{2}\left(2 g-2 m R T-3 \frac{g m^{2} \nu^{2}}{R T}\right) \\
& \bar{Y}^{\prime}=g^{2} m^{3} v\left(2 m+3 \frac{g}{R T}+g \frac{m^{2} \nu^{2}}{R T^{\prime}}\right) \\
& X X^{\prime}+\bar{Y}^{\prime} Y=\frac{g^{4} m^{6} \nu}{R T^{\prime}}\left[2\left(g-2 m R T^{\prime}+\frac{m^{2} \nu^{2}}{R T^{\prime}}\right)\left(2 g-2 m R T^{\prime}-3 \frac{g m^{9} \nu^{2}}{R T^{\prime}}\right)\right. \\
& \left.+3 g m^{2} v^{2}\left(2 m+3 \frac{g}{R T}+g \frac{m^{2} v^{2}}{R^{2} T^{2}}\right)\right] \\
& X Y^{\prime}-Y X^{\prime}=\frac{g^{2} \sqrt{g m} m^{6} v^{2}}{R T}\left[\left(g-2 m R T+\frac{g m^{2} v^{2}}{R T}\right)\left(2 m+3 \frac{g}{R T}+\frac{g^{2} m^{2} v^{2}}{R^{2} T^{2}}\right)\right. \\
& \left.-6 m\left(2 g-2 m R T-3 \frac{g m^{2} v^{2}}{R T}\right)\right] \\
& =\frac{g^{4} \sqrt{g m} m^{6} \nu^{2}}{R^{2} T^{2}}\left(3 g^{2}-15 m R T g+8 m^{2} R^{2} T^{2}+4 \frac{g m^{2} \nu^{2}}{R T}+6 g m^{3} \nu^{2}\right) \\
& \mathrm{X}^{\prime 2}+\mathrm{Y}^{\prime 2}=g^{3} m^{2}\left[4\left(2 g-2 m R T-3 \frac{g m^{2} v^{2}}{R T}\right)^{2}+g m v^{2}\left(2 m+3 \frac{g}{R T}+g \frac{m^{2} v^{2}}{R^{2} T^{2}}\right)^{2}\right]
\end{aligned}
$$

(46), (47), (48) 老組合せ 


$$
\begin{gathered}
\frac{X X^{\prime}+Y Y^{\prime}}{\mathrm{X}^{\prime 2}+Y^{\prime 2}}=\frac{g m \nu}{R T}\left[\frac{2\left(g-2 m R T^{\prime}+\frac{m^{2} \nu^{2}}{R T}\right)\left(2 g-2 m R T-3 \frac{g m^{2} \nu^{2}}{R T}\right)+3 g m \nu^{2}\left(2 m+3 \frac{g}{R T}+\frac{g m^{2} \nu^{2}}{R^{2} T^{2}}\right)}{4\left(2 g-2 m R T^{\prime}-3 \frac{g m^{2} \nu^{2}}{R T}\right)^{2}+g m \nu^{2}\left(2 m+3 \frac{g}{R T}+g \frac{m^{2} \nu^{2}}{R T^{2}}\right)^{2}}\right] \\
\frac{X Y^{\prime}-X^{\prime} Y}{X^{\prime 2}+Y^{\prime 2}}=\frac{\sqrt{g m} g m \nu^{2}}{R^{2} T^{\prime 2}}\left[\frac{3 g^{2}-15 m R T g+8 m^{2} R^{2} T^{\prime 2}+4 \frac{g m^{2} \nu^{2}}{R T}+6 g m^{3} \nu^{2}}{4\left(2 g-2 m R T-3 \frac{g m^{2} \nu^{2}}{R T}\right)^{2}+g m \nu^{2}\left(2 m+3 \frac{g}{R T}+g \frac{m^{2} \nu^{2}}{R^{\prime 2} T^{2}}\right)^{2}}\right]
\end{gathered}
$$

前述之同様 (49) と (50) は粘性の振動の振幅及び週期に及注影響を表して居る.

先最初振幅江及影響化就的て調べる.

(49) の微小項を省略する之

$$
\frac{X X^{\prime}+Y Y^{\prime}}{X^{\prime 2}+Y^{\prime 2}}=\frac{g m \nu}{R T} \frac{4(g-m R T)\left(g-2 m R T^{\prime}\right)+3 g m^{2} \nu^{2}\left(2 m+3 \frac{g}{R T}\right)}{16\left(g-m R T^{\prime}\right)^{2}+g m \nu^{2}\left(2 m+3 \frac{g}{R T}\right)}
$$

面に粘性 $\nu$ が小さければ

$$
\frac{X X^{\prime}+Y Y^{\prime}}{X^{\prime 2}+Y^{\prime 2}} \div \frac{1}{4} \frac{g m \nu}{R T} \cdot \frac{g-2 m R T}{g-m R T} \quad g \neq m R T
$$

若し $g \gg_{m} R T$ 師ち長波のものであれば（52) は

$$
\frac{X X^{\prime}+Y Y^{\prime}}{X^{2}+Y^{\prime 2}}=\frac{1}{4} \frac{g m \nu}{R T}
$$

郎ち粘性快殆ど影響しないてとてなる。

若 $g \ll m R T$ 郎亏短波長のものであれば $(52)$ は

$$
\frac{X X^{\prime}+Y Y^{\prime}}{X^{\prime 2}+Y^{\prime 2}} \div \frac{1}{2} \frac{g m \nu}{R T^{\prime}}
$$

從つて少しは数果を及卧すととが知れる。

若し $g=m R T$ ならば

$$
\frac{X X^{\prime}+Y Y^{\prime}}{X^{\prime 2}+Y^{2}}=\frac{3}{5} \frac{g^{2} y}{R^{2} T^{2}}
$$

となる。

(53)，(54) 驻省略をして居るから正式には（49）を用ひて出さねばいけない. 波動の安定不安定は

$$
\begin{array}{rlrl}
X X^{\prime}+Y Y^{\prime} & >0 & & \text { 減 塞 } \\
& = & \text { 寞 } \\
& < & & \text { 不安定 }
\end{array}
$$

を調べれてばよん。 


\section{週期に及ぼす影晦}

(50) 式の微小項を省略して

$$
\frac{X X^{\prime}-X^{\prime} Y}{\mathrm{X}^{\prime 2}+\bar{\Gamma}^{\prime 2}} \fallingdotseq \frac{\sqrt{g m} g m \nu^{2}}{R^{2} T^{\prime 2}} \cdot \frac{3 g^{2}-15 m R T g+8 m^{2} R^{2} T^{2}+6 g m^{3} \nu^{2}}{16\left(g-m R T^{\prime}\right)^{2}+g m \nu^{2}\left(2 m+3 \frac{g}{R T}\right)^{2}}
$$

若し波長汃短加い場合

$$
\frac{X Y^{\prime}-\bar{X}^{\prime} Y}{X^{\prime 2}+X^{\prime 2}} \div \frac{1}{2} \frac{\sqrt{g m} g m \nu^{q}}{R^{\prime} I^{\prime 2}}
$$

若し波長の非常に長い場合

$$
\frac{X Y^{\prime}-X^{\prime} Y}{X^{\prime 2}+Y^{\prime 2}} \doteqdot \frac{3}{16} \frac{\sqrt{g m} g m \nu^{2}}{R^{2} T^{\prime 2}} \rightarrow 0
$$

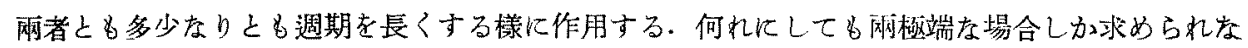
いから，本式には(50) から求めないといけない。

\section{結語}

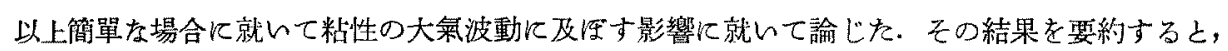
波動の振幅に及症す效果は波長の如何により減黄, 安定, 不安定振動之なる. 又粘性に無關係に 安定な振動名あり得る。週期に就いては粘性の全然なき場合よりも長くなつたり短かくなつたり する. 又粘性に無關係の場合もある. 然しながら此等の影響は若し分子粘性を用ふれば極く短波長

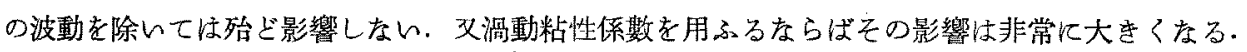
殊江短波辰の8のには著しい。

第二報では空氣の狀態變化を斷熱的艺とし一定の魀減率を有する場合について調べ倘進んで不逨 續面の場合にも應用し波動の安定，不安定を論じたいと思ふ.

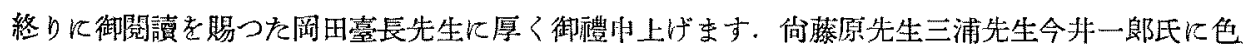
ネ指御導に與つた。茲に厚く御禮申上げをす。

\section{錄事}

\section{大日本氣呂學會講演會}

三月月次會 昭和 13 年 3 月 4 日 (金) 午後 4 時

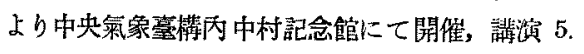

座長 传藤幹事 出席者 36 名

1. 吉野 格君：月見草の開花數と開花時刻 月見草の開花數々開化時到とについて此校的微氮

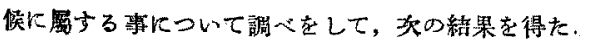

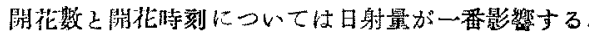

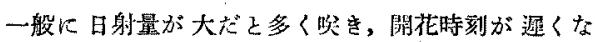
る. 花が吹くには適常な溫度があるが，そ机儿到る前 に强い日射变受ける必夏がある。

2. 山下理三郎君：大氮の振動 (荒川唀俊君代諯)

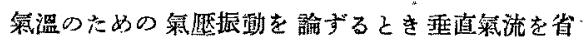

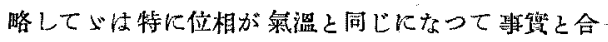
はないから之省略しないで前に一つの試みをなし 